\title{
6
}

\section{CONTINGENTES ARANCELARIOS EN LOS PRODUCTOS AGROALIMENTARIOS}

En el sector agroalimentario, en la Unión Europea, son habituales los contingentes arancelarios para establecer preferencias arancelarias.

Dada la diversidad del sector, las necesidades específicas y la evolución en los acuerdos y relaciones con otros países, se hace necesaria la existencia de diferentes tipos de contingentes. Es por esta razón que, en este artículo, se intenta dar una visión global de los contingentes que existen en el sector enmarcados en los acuerdos bilaterales y multilaterales en los que participa la UE, se aportan ejemplos de algunos de ellos y finalmente se plantean las perspectivas de futuro sobre este tema.

Palabras clave: política comercial, OMC, ventajas arancelarias, cuota, importación.

Clasificación JEL: F130, Q170, Q180.

\section{Introducción}

Los contingentes arancelarios son unas concesiones arancelarias que se dan a terceros países en los que se definen el tipo de derecho arancelario y la cantidad máxima a la que se va a aplicar. Además, según el caso, se pueden establecer condiciones específicas en su gestión con el fin de garantizar un adecuado reparto entre los operadores económicos y evitar efectos negativos sobre el mercado interno.

El artículo que se presenta tiene por objetivo dar una visión general de la casuística que

\footnotetext{
* Este artículo ha sido realizado por Sara Peña Serrano. Versión de julio de 2018.
}

existe en la actualidad en cuanto a los contingentes arancelarios en los productos agroalimentarios y su gestión en España, así como plantear los retos y perspectivas relacionados con este tema en la Unión Europea (UE) desde el punto de vista de la Administración Pública.

Para conseguir esto, el artículo se ha dividido en tres partes. En una primera parte se revisa el marco general de los contingentes, considerando aspectos en la Organización Mundial del Comercio (OMC), en el marco de los acuerdos bilaterales, y, por último, su gestión dentro de la UE.

La segunda parte presenta algunos casos de contingentes como ejemplo de la casuística descrita en la primera parte. Finalmente, se plantean algunos de los retos y perspectivas en el futuro próximo. 


\section{Tipología de contingentes agroalimentarios}

\subsection{Los contingentes y la OMC}

La Organización Mundial del Comercio debe entenderse como el marco general en el que se desarrollan las relaciones comerciales multilaterales entre los países miembros. El punto de inflexión que marca las relaciones actuales son los acuerdos como resultado de la Ronda Uruguay entre 1986 y 1994 y que dieron como resultado la OMC. De lo más general a lo particular, es preciso tomar como referencia el Acuerdo General sobre Aranceles Aduaneros y Comercio (GATT), que trata sobre el comercio de bienes, y, en particular, al Acuerdo sobre Agricultura. Este último establece compromisos en torno a tres pilares, que son el acceso a los mercados, la ayuda interna y la competencia de las exportaciones. Los contingentes arancelarios quedan bajo el paraguas de los compromisos de acceso al mercado.

Los países han consolidado sus aranceles respecto a todos los productos agropecuarios y los han consignado en las listas anexas al Acuerdo sobre la OMC. Lo mismo ha ocurrido con los contingentes. Esto quiere decir que están consolidados jurídicamente. Las listas de compromisos y el calendario de desarme de los miembros de la OMC están disponibles en la página de la OMC. Se pueden ver los primeros efectos de la Ronda Uruguay en la legislación comunitaria sobre los contingentes del sector agrícola en el Reglamento (CE) n. ${ }^{\circ} 3290 / 94$ del Consejo, de 22 de diciembre de 1994, relativo a las adaptaciones y las medidas transitorias necesarias en el sector agrícola para la aplicación de los acuerdos celebrados en el marco de las negociaciones comerciales multilaterales de la Ronda Uruguay (Unión
Europea, 1994) y en el Reglamento (CE) n.. 1808/95 del Consejo, de 24 de julio de 1995, relativo a la apertura y modo de gestión de contingentes arancelarios comunitarios consolidados en el GATT para determinados productos agrícolas, industriales y pesqueros, y a la definición de las modalidades de corrección o adaptación de los citados contingentes (Unión Europea, 1995).

La mayoría de los contingentes arancelarios de la UE consolidados ante la OMC tiene su origen en las negociaciones de la Ronda Uruguay o en los cambios sobre esas condiciones según el artículo XXVIII del GATT 1994; otros provienen de las negociaciones de adhesión a la OMC de nuevos miembros o la absorción de los contingentes que nuevos miembros de la UE tenían consolidados ante la OMC, y, en tercer lugar, existen otros contingentes que son el resultado de la aplicación de los dictámenes del órgano de solución de diferencias. Además, hay que tener en cuenta que los miembros de la OMC también pueden establecer contingentes arancelarios autónomos ${ }^{1}$ en cualquier momento, y dentro de los acuerdos de libre comercio entre la UE y terceros países, en los que las concesiones pueden darse en forma de contingentes.

Dentro del Acuerdo de Agricultura, los miembros están obligados a hacer notificaciones sobre compromisos dentro del acuerdo (artículo 18.2) y, en particular, en cuanto a los contingentes arancelarios. Esto se hace en los Cuadros MA.1 y MA.2, en los que se notifica la tasa de utilización de los mismos. El cumplimiento de los compromisos en el marco de $\triangleright$

\footnotetext{
1 En la actualidad, los contingentes autónomos se regulan por el Reglamento (UE) n.․ 1388/2013 del Consejo, de 17 de diciembre de 2013, relativo a la apertura y modo de gestión de contingentes arancelarios autónomos de la Unión para determinados productos agrícolas e industriales y por el que se deroga el Reglamento (UE) n. 0 7/2010 y modificaciones posteriores. Hay contingentes para granos de mazorca, setas, cerezas y tabaco en rama.
} 
la OMC y el uso de prácticas leales y que no distorsionen el comercio es una de las cuestiones principales que se está demandando últimamente en la OMC; así, por ejemplo, EEUU, Japón y la UE hicieron una declaración conjunta en la última Conferencia Ministerial, en 2017, en Buenos Aires (Malmström, C.; Seko, H. y Lighthizer, R.E., 2017). Cumplir con los compromisos relativos a las notificaciones a la OMC de los Estados miembros y que mejoran la transparencia es crucial.

En particular, para la UE hay 124 contingentes agrícolas que están comprometidos en el marco de la OMC. Estos se separan en dos grupos, aquellos que cubren el año civil, que son 64 , y los que se aplican durante la campaña de comercialización, que son 60 (OMC, 2018) ${ }^{2}$. De todos ellos, algo más de 40 se usan por encima del 90 por 100; unos 20, entre el 25 y el 90 por 100, y el resto se usan poco o nada. Entre los contingentes muy demandados, se puede destacar el de carne de vacuno, de 53.000 toneladas $(t)$, algunos de los contingentes de carne de ave, algunos contingentes de frutas y hortalizas, el contingente de almendra, algunos contingentes de arroz o los contingentes de maíz específicos para España y Portugal (2.000.000 t y 500.000 t respectivamente), entre otros. De los contingentes que se usan menos del 90 por 100 se pueden destacar el de carne de caprino y ovino, de $282.390 \mathrm{t}$, el contingente de ajo de $71.245 \mathrm{t}$, dos contingentes para cítricos (minneolas, $15.000 \mathrm{t}$ y limones $10.000 \mathrm{t}$ ) o el contingente de $15.005 \mathrm{t}$ de queso cheddar.

Por último, hay 64 contingentes que se usan poco o nada. Algunos de estos son los contingentes de animales vivos de ganado vacuno,

2 OMC (2018). Última notificación de la UE al Comité de Agricultura de la OMC, G/AG/N/EU/33, correspondiente a las campañas de comercialización 2014/2015 y 2015/2016 y al año civil 2015. chocolate, cebada, conservas de champiñones o vermut.

El grado de utilización de los contingentes depende de la demanda en la UE de esos productos, del volumen del contingente y de las ventajas arancelarias que ofrece frente a otros o al arancel aplicado al país.

En relación a la administración de los contingentes arancelarios, en la Conferencia Ministerial de 2013, en Bali, se llegó a un entendimiento relativo a las disposiciones sobre la administración de los productos agropecuarios, según el artículo 2 del Acuerdo sobre Agricultura (OMC, 2013). Una de las cuestiones que se consideran es que la administración de los contingentes constituye un caso de «trámite de licencias de importación» y, por tanto, es aplicable el Acuerdo sobre Procedimientos para el Trámite de Licencias de Importación de la Ronda Uruguay (OMC, 2013). Es por ello que al Comité de licencias de importación de la OMC se notifican anualmente los procedimientos sobre licencias de importación de acuerdo con el artículo 7.3 del acuerdo, ya que estas son necesarias para determinados productos agrícolas importados en el marco de contingentes arancelarios ${ }^{3}$. En esta notificación se explica la legislación aplicable, el procedimiento general y, para cada sector, el fundamento jurídico y el alcance.

\subsection{Los contingentes y los acuerdos de libre comercio}

En los acuerdos de libre comercio, las concesiones arancelarias se materializan en reducciones o eliminación de los aranceles y, $\triangleright$

3 OMC (2017). Respuesta al cuestionario sobre los procedimientos para el trámite de licencias de importación. Notificación en virtud del párrafo 3 del artículo 7 del acuerdo sobre procedimientos para el trámite de licencias de importación. Unión Europea. G/LIC/3/EU/6. 
TABLA 1

EJEMPLOS DE CONTINGENTES NEGOCIADOS EN LOS ACUERDOS ENTRE LA UE Y TERCEROS PAÍSES

\begin{tabular}{|c|c|c|}
\hline País & Estado del acuerdo & Contingentes de la UE negociados en los acuerdos \\
\hline Canadá & $\begin{array}{l}\text { Aplicado de forma } \\
\text { provisional desde el } \\
21 \text { de septiembre de } \\
2017\end{array}$ & $\begin{array}{l}\text { Camarones transformados, bacalao congelado y el trigo blando de calidad media y baja, } \\
\text { maíz dulce, bisonte, carne de vacuno fresca o refrigerada, carne de vacuno congelada y las } \\
\text { demás carnes de vacuno, carne de porcino }\end{array}$ \\
\hline Chile & $\begin{array}{l}\text { Acuerdo en vigor } \\
\text { desde } 2003\end{array}$ & $\begin{array}{l}\text { Carne de bovino, carne y productos del porcino, carne de ovino-caprino, carne de ave; } \\
\text { quesos, ajos, uvas, arroz, hongos, cerezas; artículos de confitería, chocolate, productos de } \\
\text { panadería, pastelería o galletería, pescado fresco, pescado seco, salado o en salmuera, } \\
\text { atún en conserva }\end{array}$ \\
\hline Egipto & $\begin{array}{l}\text { Acuerdo de } \\
\text { asociación en vigor } \\
\text { desde } 2004\end{array}$ & $\begin{array}{l}\text { Naranjas, bulbos, plantas, flores, otras plantas, patatas, cebollas, ajos, coles, lechuga, } \\
\text { zanahorias, pepinos, legumbres, hongos, frutas desecadas, patatas, melones, peras, } \\
\text { melocotón, ciruelas, fresas, frutos secos, arroz, aceite de sésamo, otros aceites vegetales, } \\
\text { melaza, mermeladas, cacahuetes, zumos de fruta }\end{array}$ \\
\hline Marruecos & $\begin{array}{l}\text { Acuerdo en vigor } \\
\text { desde marzo de } \\
2000\end{array}$ & $\begin{array}{l}\text { Tomates, alcachofas, pepinos, clementinas, naranjas, flores, patatas, cebollas, coles, } \\
\text { lechuga iceberg, calabacines, cebollas salvajes, varias hortalizas, hortalizas congeladas, } \\
\text { membrillos, pepinillos preparados o conservados, guisantes y judías verdes, pulpa de } \\
\text { albaricoques, mezcla de frutas, jugo de naranja, vino, preparaciones y conservas de } \\
\text { sardinas }\end{array}$ \\
\hline México & $\begin{array}{l}\text { Acuerdo en vigor } \\
\text { desde } 2000\end{array}$ & $\begin{array}{l}\text { Huevos con cáscara, huevos sin cáscara, miel, flores, espárragos, guisantes, fresas, } \\
\text { conservas de atún, melaza, conservas de espárrago, conservas de palmito, zumo de } \\
\text { naranja, zumo de piña, albúminas }\end{array}$ \\
\hline $\begin{array}{l}\text { Sudáfrica } \\
\text { (como parte } \\
\text { de la SADC) }\end{array}$ & $\begin{array}{l}\text { Firmado en junio de } \\
2016 \text {, operativo en } \\
\text { febrero de } 2018\end{array}$ & $\begin{array}{l}\text { Leche desnatada en polvo, mantequilla, flores: rosa, orquídea y crisantemo; lilas y otros, } \\
\text { flores no frescas, fresas, azúcar, glucosa en polvo cristalino blanco, mermeladas de cítricos, } \\
\text { fruta conservada de peras, albaricoques y melocotones, y mezclas de frutas no tropicales, } \\
\text { fruta tropical envasada, zumo de naranja concentrado, zumo de manzana y zumo de piña, } \\
\text { levaduras vivas, vinos y etanol }\end{array}$ \\
\hline Turquía & $\begin{array}{l}\text { Unión aduanera } \\
\text { desde } 1995\end{array}$ & $\begin{array}{l}\text { Carne de pavo, queso, cebollas, berenjenas, calabacines, sandías, fresas y frambuesas } \\
\text { congeladas, conservas de tomate, pulpa de albaricoque, vacuno }\end{array}$ \\
\hline Ucrania & $\begin{array}{l}\text { Acuerdo en vigor } \\
\text { desde enero de } \\
2016\end{array}$ & $\begin{array}{l}\text { Carnes: vacuno, porcino, ovino y ave; leche, nata y yogures, leche en polvo, mantequilla, } \\
\text { huevos y albúminas, miel, azúcares, trigo blando, harina y pellets, cebada, avena, maíz, } \\
\text { grañones y sémola de cebada, malta y gluten de trigo, almidón y fécula, almidón y fécula } \\
\text { transformados, salvado, moyuelos y residuos, hongos, tomates transformados, jugo de uva } \\
\text { y manzana, productos transformados a base de leche transformada, productos a base de } \\
\text { mantequilla transformada, maíz dulce, productos transformados a base de azúcar, } \\
\text { productos transformados a base de cereales, productos transformados a base de nata, } \\
\text { preparaciones alimenticias, etanol, cigarrillos, manitol-sorbitol y productos transformados a } \\
\text { base de almidón o fécula de malta }\end{array}$ \\
\hline Vietnam & $\begin{array}{l}\text { Negociaciones } \\
\text { terminadas en } \\
\text { diciembre de } 2016\end{array}$ & $\begin{array}{l}\text { Huevos y yemas, ajo, maíz dulce, arroz, fécula de mandioca, conservas de atún, surimi, } \\
\text { azúcar, champiñones, etanol }\end{array}$ \\
\hline \multicolumn{3}{|c|}{ Fuente: elaboración propia con datos de los textos de los acuerdos con terceros países publicados en el Diario Oficial de la UE. } \\
\hline
\end{tabular}

en algunos productos, las concesiones quedan limitadas a contingentes arancelarios en los que se especifica la cantidad del contingente, arancel y modo de gestión. Estos contingentes suelen establecerse en aquellos productos que la UE no quiere liberalizar totalmente.

En la tabla se muestran algunos ejemplos de las concesiones en forma de contingentes que la UE ha dado en diferentes acuerdos que tiene firmados con terceros países (se han tomado como referencia los primeros acuerdos suscritos, sin considerar las renovaciones 0 ampliaciones de concesiones posteriores). Se puede observar que las concesiones mediante contingentes suelen ser especialmente para los productos con mayor protección arancelaria dentro de la UE, como algunas frutas y hortalizas, carnes, lácteos, etcétera.

Los contingentes no suelen ser estáticos, y estos pueden variar a lo largo del tiempo en $\square$ 
su modo de gestión, así como las concesiones arancelarias que en ellos se dan. En los acuerdos de libre comercio se suelen acordar las concesiones de partida con posibles incrementos en las cantidades concedidas, o con posibles reducciones arancelarias a las cantidades asignadas en el contingente. A medida que las relaciones con el tercer país van avanzando, se pueden establecer nuevas concesiones.

El modo en que estas concesiones se hacen es variado. Por ejemplo, con Islandia (Unión Europea, 2017b) o Noruega (Unión Europea, 2018) se han materializado las concesiones en la forma de canjes de notas, mientras que en el caso de México, durante los años 2017 y 2018 la Comisión ha estado negociando la renovación del acuerdo de libre comercio, llegando a un acuerdo de principio en abril de 2018 y en el que se ha buscado ampliar el alcance de las concesiones arancelarias entre la UE y México (Comisión Europea, 2018).

Por otro lado, el nivel de detalle en la gestión de un contingente varía y, así, por ejemplo, hay en algunos acuerdos en los que se limita a describir cantidades, aranceles y plazos de escalonamiento, mientras que en otros se llegan a describir condiciones en el modo de gestión del continente, como es el caso del acuerdo con Canadá, en el que se incluye una declaración relativa a la administración, por parte de Canadá, de los contingentes arancelarios de queso.

\subsection{Los contingentes en la UE y su gestión}

El Código Aduanero de la Unión compendia la legislación básica en cuestiones arancelarias en la UE. Está establecida en el Reglamento (UE) n. 9 952/2013 del Parlamento Europeo y del Consejo, de 9 de octubre de 2013, por el que se establece el código aduanero de la Unión (Unión Europea, 2013b), y en las normas de desarrollo del mismo, que vienen recogidas en el Reglamento de Ejecución (UE) 2015/2447 de la Comisión, de 24 de noviembre de 2015, por el que se establecen normas de desarrollo de determinadas disposiciones del Reglamento (UE) n. ${ }^{\circ}$ 952/2013 del Parlamento Europeo y del Consejo, por el que se establece el código aduanero de la Unión (Unión Europea, 2015b).

En cuanto a cuestiones relacionadas con los contingentes arancelarios es preciso referirse a esta legislación, en particular al artículo 56 del Código Aduanero Común, que trata sobre el arancel aduanero común y vigilancia, y en los artículos 49 al 54 del Reglamento (UE) $2015 / 2447$, en los que se habla de la gestión de los contingentes arancelarios. Algunas de las características sobre estos contingentes son:

- Se gestionan según el orden cronológico de llegada.

- Cada contingente arancelario se identifica mediante un número de orden.

- Las autoridades aduaneras de los Estados miembros (EEMM) tienen responsabilidades con el fin de llevar a cabo una gestión uniforme de los contingentes y se refieren al examen de la solicitud y a la comunicación con la Comisión Europea.

- La Comisión Europea es la encargada de asignar las cantidades.

En el ámbito de la agricultura también existe base legislativa relativa a los contingentes arancelarios, y para ello es preciso revisar el Reglamento (UE) n. ${ }^{\circ}$ 1308/2013 del Parlamento Europeo y del Consejo, de 17 de diciembre de 2013, por el que se crea la organización $\triangleright$ 
común de mercados de los productos agrarios y por el que se derogan los Reglamentos (CEE) n. $-922 / 72$, (CEE) n.․ 234/79, (CE) n. $.1037 / 2001$ y (CE) n.o 1234/2007 (Unión Europea, 2013a), que recoge, en el capítulo III de la parte III relativa a los intercambios comerciales con terceros países, cuestiones sobre la gestión de los contingentes arancelarios y regímenes especiales de importación por terceros países.

En este reglamento se identifican los métodos principales de gestión de los contingentes arancelarios. El primero de ellos es el método basado en el orden cronológico de presentación de llegada o principio de «orden de llegada", el segundo es el método de prorrateo en función de las cantidades solicitadas o método de «examen simultáneo», y el tercero es el método basado en las corrientes comerciales tradicionales o método denominado «tradicionales/recién llegados». En el artículo 185 existe una mención a contingentes arancelarios específicos y hace referencia expresa a unos contingentes arancelarios de importación de 2.000 .000 toneladas de maíz y 300.000 toneladas de sorgo para España y 500.000 toneladas de maíz para Portugal. En los artículos 187 y 188 se describen las competencias de ejecución atribuidas a la Comisión, entre los que están que la Comisión puede adoptar los actos de ejecución en los que se establecen las normas de aplicación de los contingentes.

En cuanto al primer método de gestión que identifica la legislación horizontal de agricultura, la gestión suele llevarse a cabo por las autoridades aduaneras de los EEMM, y en la Comisión Europea, por la Dirección General de Fiscalidad y Unión Aduanera (DG TAXUD), mientras que en los otros dos métodos, tanto el de examen simultáneo como el de tradicionales/recién llegados, se suele exigir la emisión de un certificado de importación, que se conoce como certificado AGRIM. En este caso, la gestión a nivel de la Comisión es realizada por la Dirección General de Agricultura y Desarrollo Rural (DG AGRI), y en España, la emisión de certificados de importación (y de exportación) en el comercio exterior de productos agroalimentarios es competencia de la Subdirección General de Comercio Internacional de Productos Agroalimentarios.

Para conocer el nivel de uso de los contingentes hay que atender a estas cuestiones, ya que la información la maneja o bien la DG TAXUD o bien DG AGRI. En cuanto a la información de los contingentes gestionados por el método de primer llegado, primer servido, la información sobre el grado de utilización puede encontrarse en la página web http://ec.europa.eu/taxation_customs/dds2/taric/quota consultation.jsp? Lang=en. Mientras que en el caso de los contingentes, cuya información se gestiona desde DG AGRI, está disponible la información sobre los coeficientes de asignación, de modo que si el coeficiente de asignación es menor a 100, se entenderá que se ha solicitado toda la cantidad disponible para el periodo del que se trate, y, por tanto, salvo circunstancias especiales, se usará en su totalidad. Estos coeficientes se publican periódicamente en la página web https://ec.europa.eu/agriculture/tariff-rate-quotas-trqs_en

Además de los tipos generales descritos, se pueden encontrar más variables que generan diferencias entre los distintos tipos de contingentes. En particular, dentro del grupo de los contingentes gestionados por «examen simultáneo» nos encontramos con los siguientes tipos:

- Documentos emitidos por el tercer país (certificado de exportación, certificado de autenticidad, etcétera). 
- Asignación de derechos de importación y posterior emisión de certificados.

- Solicitud de certificados y prorrateo de las cantidades.

Por otro lado, existe una serie de normas comunes en la gestión de certificados a nivel horizontal ${ }^{4}$ y a nivel sectorial, además de las particularidades de cada contingente, y que generan diferencias entre los contingentes ${ }^{5}$, como pueden ser:

- Gestión de los periodos contingentarios (anual, trimestral, mensual).

- Validez de los derechos (anual, trimestral, hasta el final del contingente).

- Condiciones de acceso al contingente (prueba de comercio).

- Condiciones sobre la cantidad que puede solicitar un operador (cantidad de referencia, porcentaje mínimo y/o máximo sobre la cantidad disponible).

- Validez de los certificados (mensual, trimestral, hasta el final del periodo contingentario, etcétera).

\section{Casos específicos}

Una vez revisado el marco general en el que se encuadran los contingentes arancelarios del sector agroalimentario, se van a explicar brevemente algunas de las características

4 Reglamento de ejecución (UE) 2016/1239 de la Comisión, de 18 de mayo de 2016, por el que se establecen disposiciones de aplicación del Reglamento (UE) n.ำ 1308/2013 del Parlamento Europeo y del Consejo en lo relativo al régimen de certificados de importación y exportación (Unión Europea, 2016)

5 En la Circular de 3 de abril de 2014, de la Dirección General de Comercio e Inversiones, relativa al procedimiento y tramitación de las importaciones e introducciones de mercancías y sus regímenes comerciales (Ministerio de Economía y Competitividad, 2014) viene recopilada la información básica actualizada en el momento de su publicación. de contingentes que pueden encontrarse en la actualidad con el fin de mostrar la diversidad de los mismos por su origen, modo de gestión o condiciones.

\subsection{Contingentes de carne de ave procedente de Brasil, Tailandia $y$ otros países}

Estos contingentes son consecuencia de una disputa ante la OMC entre Brasil y la UE por una reclasificación arancelada de la carne salada. Este tipo de carne accedía a la UE con un arancel más bajo previo a la modificación y se entendió que era necesario hacer una compensación a los países exportadores, que en ese caso eran Brasil y Tailandia. Como consecuencia de esta disputa, se han desarrollado varios contingentes que se recogen en dos reglamentos:

- Reglamento (CE) n.ำ 1385/2007 de la Comisión, de 26 de noviembre de 2007, por el que se establecen disposiciones de aplicación del Reglamento (CE) 774/94 del Consejo en lo que concierne a la apertura y modo de gestión de determinados contingentes arancelarios comunitarios en el sector de la carne de aves de corral (Unión Europea, 2007a).

- Reglamento (CE) n.o 616/2007 de la Comisión, de 4 de junio de 2007, relativo a la apertura y modo de gestión de contingentes arancelarios comunitarios en el sector de la carne de aves de corral originaria de Brasil, Tailandia y otros terceros países (Unión Europea, 2007c).

Si atendemos al Reglamento (CE) 1385/2007, el sistema de gestión se realiza mediante la $\triangle$ 
solicitud de emisión de certificados y prorrateo de las cantidades. El contingente es anual y se divide en cuatro subperiodos. Además, la cantidad disponible se distribuye por países y por productos. Hay seis grupos, tres para carne de pollo y tres para carne de pavo, y cantidades específicas asignadas a Brasil, Tailandia y otros países. El contingente exige la presentación de un certificado AGRIM, y establece algunas condiciones para poder aceptar la solicitud que son una prueba de comercio en los dos años anteriores, y exige una cantidad mínima y máxima que puede solicitar cada operador. En los certificados además establece algunas condiciones relativas al periodo de validez, que es de 150 días desde el primer día del inicio del subperiodo al que se refieran.

Atendiendo al Reglamento (CE) 616/2007, la gestión de algunos grupos se realiza mediante la solicitud de emisión de certificados y prorrateo de las cantidades y, en dos de los grupos, la gestión se lleva a cabo mediante la asignación de derechos de importación y expedición posterior de los certificados de importación. Los contingentes son anuales para todos los grupos y presentan división en subperiodos en algunos de los grupos.

Hay once grupos, tres de ellos para carne salada de Brasil, Tailandia y otros países, seis grupos para preparaciones de carne de ave, de los que hay dos por cada país, y otros dos grupos para preparaciones de carne de pavo con una periodicidad trimestral o anual. En todos ellos se exige prueba de comercio y se establecen cantidades mínimas y máximas que pueden hacerse por solicitud. En cuanto a la validez de los certificados y de los derechos, estos son válidos hasta el final del periodo contingentario, salvo en los dos grupos gestionados con derechos de importación, para los que los certificados tienen una validez de quince días.

\subsection{Contingente de ajo}

Este contingente está comprometido ante la OMC, y merece especial mención por dos razones: en primer lugar porque el método de gestión se basa en el método de tradicionales y nuevos importadores y porque se trata de un producto de interés para España. Estos contingentes se regulan por el Reglamento (CE) n. ${ }^{\circ} 341 / 2007$ de la Comisión, de 29 de marzo de 2007 , por el que se abren contingentes arancelarios, se fija su modo de gestión y se instaura un régimen de certificados de importación y de origen para los ajos importados de terceros países (Unión Europea, 2007b).

El contingente es anual, y las cantidades disponibles se dividen en cuatro subperiodos. Se trata de seis números de orden que se corresponden con dos por cada origen (Argentina, China y otros terceros países) y desglosados por importadores tradicionales y nuevos importadores. El 70 por 100 del mismo se destinará a operadores tradicionales y el 30 por 100 , a nuevos operadores.

En cuanto a la solicitud de certificados, los importadores tradicionales pueden solicitar como máximo la cantidad de referencia (con base en la media importada de los tres años anteriores), y en el caso de los importadores nuevos será como máximo del 10 por 100 de la cantidad establecida para el periodo contingentario. Una vez se hacen las solicitudes de certificados, se prorratean las cantidades $y$ posteriormente se emiten.

\subsection{Contingente de huevos de Ucrania}

Este contingente nace como consecuencia de concesiones arancelarias de la UE a Ucrania dentro del Acuerdo de Asociación $\triangleright$ 
entre la UE y Ucrania de 2014. Las normas del contingente se rigen por el Reglamento de ejecución (UE) 2015/2077 de la Comisión, de 18 de noviembre de 2015, relativo a la apertura y modo de gestión de contingentes arancelarios de importación de la Unión de huevos, ovoproductos y albúminas originarios de Ucrania (Unión Europea, 2015 a).

La gestión se realiza mediante la emisión de certificados y posterior prorrateo de las cantidades, de acuerdo al reparto trimestral del contingente. Para este contingente existe una cantidad mínima y máxima que se puede hacer de las solicitudes, y los certificados tendrán una validez de 150 días desde su expedición el primer día del subperiodo para el que se expidan.

\subsection{Contingentes de carne de vacuno}

Dentro del sector de la carne de vacuno se encuentran distintos contingentes y con diferentes modos de gestión. En primer lugar, se puede hablar del contingente regulado por el Reglamento de Ejecución (UE) n. 481/2012 de la Comisión, de 7 de junio de 2012, por el que se establecen las normas de gestión de un contingente arancelario de carne de vacuno de calidad superior (Unión Europea, 2012), que da respuesta a una disputa entre EEUU y la UE por la carne hormonada, y se gestiona mediante primer llegado, primer servido a través de las autoridades aduaneras. Este contingente exige la presentación de un certificado de autenticidad a las autoridades aduaneras para poder llevar a cabo el despacho.

Un segundo grupo de contingentes dentro del sector de la carne de vacuno son los que se regulan por el Reglamento de Ejecución (UE) n. ${ }^{\circ}$ 593/2013 de la Comisión, de 21 de junio de
2013, relativo a la apertura y el modo de gestión de los contingentes arancelarios de carnes de vacuno de calidad superior fresca, refrigerada o congelada, y de carne de búfalo congelada (Unión Europea, 2013c), y por el Reglamento (CE) n. $610 / 2009$ de la Comisión, de 10 de julio de 2009, por el que se establecen las disposiciones de aplicación del contingente arancelario de carne de vacuno originaria de Chile (Unión Europea, 2009). En la mayoría de los números de orden incluidos en estos reglamentos es necesaria la presentación de un certificado de autenticidad previo a la emisión del certificado AGRIM.

El tercer contingente destacable es el que se regula mediante el Reglamento (CE) 431/2008 de la Comisión, de 19 de mayo de 2008, relativo a la apertura y modo de gestión de un contingente arancelario de importación de carne de vacuno congelada del código NC 0202 y productos del código NC 02062991 (Unión Europea, 2008b), y que nace como consecuencia de las obligaciones de la UE ante la OMC, y se gestiona mediante la asignación de derechos en un primer momento y posterior emisión de certificados. La diferencia con otros contingentes es que, en este caso, el contingente es anual, no tiene subperiodos y establece como cantidad máxima para la asignación de derechos una «cantidad de referencia».

\subsection{Contingente de aceite procedente de Túnez}

Este contingente es una concesión específica de la UE a Túnez que se encuentra enmarcada en el Acuerdo Euromediterráneo en la asociación entre Túnez y la UE y regula sus condiciones en el Reglamento (CE) n.ำ1918/2006 de la Comisión, de 20 de diciembre de 2006, relativo a la apertura y gestión de un contingente $\triangleright$ 
arancelario de aceite de oliva originario de Túnez (Unión Europea, 2006a).

Este contingente tiene muy pocas restricciones comparado con otros a la hora de establecer las condiciones de acceso de los operadores. Es un contingente anual del que se pueden hacer solicitudes de certificados semanalmente, y una vez que se emiten tienen una duración hasta el final del periodo. Por este motivo, este contingente suele agotarse al inicio del mismo en términos de solicitud de certificados y prorrateo de las cantidades, si bien las importaciones efectivas se van espaciando a lo largo del año.

\subsection{Contingentes de arroz}

En el caso de los contingentes de arroz, varios de ellos están regulados por el Reglamento de Ejecución (UE) n.ำ1273/2011 de la Comisión, de 7 de diciembre de 2011, relativo a la apertura y el modo de gestión de determinados contingentes arancelarios de importación de arroz y arroz partido (Unión Europea, 2011). Se trata de una serie de contingentes para la importación de arroz partido, descascarillado y blanqueado cuyas cantidades se distribuyen entre varios países. Son contingentes que están comprometidos ante la OMC y que se gestionan mediante la solicitud de certificados y posterior prorrateo de las cantidades. A diferencia de otros contingentes, en el caso de las importaciones procedentes de Tailandia, Australia y EEUU, en la solicitud de certificado de importación se exige la presentación de un certificado de exportación.

\subsection{Contingentes de maíz y sorgo}

Por último, se mencionan brevemente los contingentes arancelarios para la importación de maíz y sorgo de España y maíz de Portugal. Estos contingentes existen derivados de compromisos de España y Portugal anteriores a la entrada en la Comunidad Económica Europea y que en el marco de las negociaciones comerciales multilaterales de la Ronda Uruguay la Comunidad se comprometió a abrir a partir de la campaña de comercialización 1995/96. En particular son 500.000 toneladas de maíz a Portugal y 2.000.000 de toneladas de maíz y 300.000 toneladas de sorgo en España. Estos contingentes tienen un funcionamiento particular y se regulan por el Reglamento (CE) $n$. o 1296/2008 de la Comisión, de 18 de diciembre de 2008, por el que se establecen disposiciones de aplicación de los contingentes arancelarios por importación de maíz y de sorgo en España y de maíz en Portugal (Unión Europea, 2008a).

\section{Retos y perspectivas de futuro}

En este artículo se ha pretendido dar una visión general de la casuística existente en la gestión de contingentes arancelarios con algunas referencias concretas de los mismos.

De cara al futuro, la gestión de los contingentes se enfrenta en la actualidad a varios retos. En primer lugar, estos se ven afectados por la evolución de las concesiones comerciales siguiendo la tendencia de la UE hacia una mayor liberalización comercial. En segundo lugar, por los procedimientos internos de la UE hacia una racionalización y simplificación de la legislación europea y, en tercer lugar, por las consecuencias de la salida de la UE de Reino Unido sobre los compromisos bilaterales y multilaterales de la UE.

La Unión Europea negocia acuerdos de libre comercio con terceros países. Esto implica $\triangleright$ 
directamente que se hagan concesiones, que toman la forma de contingentes en aquellos casos en los que se quiere limitar la cantidad de producto. Por tanto, el número de contingentes aumentará a medida que se firmen nuevos acuerdos. Por otro lado, los acuerdos no son estáticos, los contingentes pueden ir aumentando el volumen o reduciendo los aranceles, o incluso desaparecer si se convierten en una liberalización de la partida afectada. La realidad es que en aquellos productos en los que no se quiera hacer una liberalización total, esta puede quedar en forma de contingente.

En cuanto a la legislación de la Unión Europea, dentro de la Organización Común de Mercados Única, ya se unificaron las diferentes organizaciones comunes de mercados en un solo reglamento, y la misma idea existe para la gestión de contingentes. Así, los EEMM llevan negociando desde el año 2014 un Reglamento Delegado y un Reglamento de Ejecución común para la gestión de contingentes arancelarios gestionados mediante licencias. En estos reglamentos se incluirían las disposiciones comunes a todos los contingentes gestionados por certificados y las particularidades de cada uno de ellos, respondiendo a las necesidades del mercado. Esto, previsiblemente, supondrá cambios en la forma de gestión, simplificando algunos contingentes y modificando algunas variables de otros.

Por último, la salida de Reino Unido de la Unión Europea afecta también a los contingentes. Dado que Reino Unido ya no formará parte de la Unión Aduanera, se hace necesario negociar en relación a los contingentes, y muy en particular, aquellos que se encuentran comprometidos ante la OMC. Actualmente las mercancías pueden ser despachadas en un punto de inspección de un país y ser consumidas en otro Estado miembro. Las empresas pueden estar radicadas en un país desde el que centralicen todas las operaciones aduaneras y posteriormente distribuir la mercancía por la Unión Europea. Esto es importante, ya que el uso de los contingentes arancelarios por los EEMM no refleja el consumo real de esos productos en esos EEMM. De modo que la Comisión Europea se enfrenta al reto de negociar con Reino Unido de qué manera hacer el reparto de estos contingentes y hacerlo de modo que sea compatible con los compromisos ante la OMC.

\section{Bibliografía}

[1] COMISIÓN EUROPEA (2016). EU-Vietnam Free Trade Agreement: agreed text as of January 2016. Disponible en: http://trade. ec.europa.eu/doclib/press/index.cfm?id=1437

[2] COMISIÓN EUROPEA (2018). EU and Mexico reach a new agreement on trade. Nota de prensa, 21 de abril de 2018. Disponible en: http://trade.ec.europa.eu/doclib/press/index. $c f m ? i d=1830$

[3] MALMSTRÖM, C.; SEKO, H. y LIGHTHIZER, R.E. (2017). Joint statement. Disponible en: http://trade.ec.europa.eu/doclib/docs/2017/ december/tradoc_156458.pdf

[4] MINISTERIO DE ECONOMÍA Y COMPETITIVIDAD (2014). Circular de 3 de abril de 2014, de la Dirección General de Comercio e Inversiones, relativa al procedimiento y tramitación de las importaciones e introducciones de mercancías y sus regímenes comerciales (BOE, n. .88 de 11 de abril de 2014, p. 3010530182).

[5] OMC (2013). Understanding on tariff rate quota administration provisions of agricultural products, as defined in article 2 of the agreement on agriculture, Decisión Ministerial WT/ $M I N(13) / 39$. En: Ministerial Declaration and Decisions, Ministerial Conference ninth session. Bali, 3-6 december 2013. Disponible en: https://www.wto.org/english/thewto_e/minist_elmc9_e/balideclaration_e.htm 
Subdirección General de Comercio Internacional de Productos Agroalimentarios

[6] OMC (2018). Notificación: G/AG/N/EU/41. Disponible en: https://docs.wto.org/dol2fe/ Pages/FE_Search/FE_S_S009-DP.aspx?language $=E \&$ Catalogueld List $=243355,243265$, 242010,241964,241912,241829,241616, 241615,241612,240409\&CurrentCatalogue IdIndex $=3 \&$ FullTextHash $=\&$ HasEnglish Record $=$ True\&HasFrenchRecord $=$ True\&Has SpanishRecord $=$ True

[7] UNIÓN EUROPEA (1994). «Reglamento (CE) n.. 3290/94 del Consejo, de 22 de diciembre de 1994, relativo a las adaptaciones y las medidas transitorias necesarias en el sector agrícola para la aplicación de los acuerdos celebrados en el marco de las negociaciones comerciales multilaterales de la Ronda Uruguay». Diario oficial, n.ํ L 349 de 31 de diciembre de 1994, pp. 105-200. Disponible en: https://eur-lex.europa.eu/legal-content/ES/ $T X T /$ ? qid $=1528458917868$ \& $u r i=C E L E X$ : $31994 R 3290$

[8] UNIÓN EUROPEA (1995). «Reglamento (CE) n. $.91808 / 95$ del Consejo de 24 de julio de 1995 relativo a la apertura y modo de gestión de contingentes arancelarios comunitarios consolidados en el GATT para determinados productos agrícolas, industriales y pesqueros, y a la definición de las modalidades de corrección o adaptación de los citados contingentes». Diario oficial, n.. $\mathrm{L} 176$ de 27 de julio de 1995, pp. 1-91. Disponible en: https://eur-lex. europa.eu/legal-content/ES/TXT/?qid= 1528459929779\&uri=CELEX:31995R1808

[9] UNIÓN EUROPEA (1998). «Protocolo n. 1 relativo al régimen preferencial aplicable por la Comunidad a la importación de productos agrícolas originarios de Turquía». Diario oficial, n. ${ }^{\circ}$ L86 de 20 de marzo de 1998, pp. 3-8.

[10] UNIÓN EUROPEA (2000). «Decisión N N.o 2/2000 del Consejo Conjunto CE-México de 23 de marzo de 2000». Diario oficial, n. ${ }^{\circ}$ L157 de 30 de junio de 2000, pp. 10-29.

[11] UNIÓN EUROPEA (2002). «Acuerdo por el que se establece unaAsociación entre la Comunidad Europea y sus Estados miembros, por una parte, y la República de Chile, por otra». Diario oficial, n.. L352 de 30.12.2002, pp. 3/1450.

[12] UNIÓN EUROPEA (2004). «Acuerdo Euromediterráneo por el que se establece una Asociación entre las Comunidades Europeas y sus Estados miembros, por una parte, y la República Árabe de Egipto, por otra». Diario oficial, n. ${ }^{\circ}$ L304 de 30 de septiembre de 2004, pp. 39-209.

[13] UNIÓN EUROPEA (2006a). «Reglamento (CE) n.o 1918/2006 de la Comisión de 20 de diciembre de 2006 relativo a la apertura y gestión de un contingente arancelario de aceite de oliva originario de Túnez». Diario oficial, n. ${ }^{\circ} 365$ de 21 de diciembre de 2006. Disponible la última versión consolidada en: https://eur-lex.europa.eu/legal-content/ES/ $T X T /$ ? qid $=1528360460693$ \& uri $=$ CELEX: 02006R1918-20160427

[14] UNIÓN EUROPEA (2007a). Reglamento (CE) n.. 1385/2007 de la Comisión de 26 de noviembre de 2007 por el que se establecen disposiciones de aplicación del Reglamento (CE) n.. 774/94 del Consejo en lo que concierne a la apertura y modo de gestión de determinados contingentes arancelarios comunitarios en el sector de la carne de aves de corral. Diario oficial n.. L309 de 27 de noviembre de 2007, p 47-55. Disponible en: https://eur-lex. europa.eu/legal-content/ES/TXT/?qid= $1528382230607 \& u r i=C E L E X: 32007 R 1385$

[15] UNIÓN EUROPEA (2007b). «Reglamento (CE) n.․ 341/2007 de la Comisión, de 29 de marzo de 2007, por el que se abren contingentes arancelarios, se fija su modo de gestión y se instaura un régimen de certificados de importación y de origen para los ajos importados de terceros países». Diario oficial, n. ㄴ L90 de 30 de marzo de 2007. Disponible la última versión consolidada en: https://eur-lex.europa.eu/legal-content/ES/TXT/?qid $=152835$ 8565291\&uri=CELEX:02007R0341-20170101

[16] UNIÓN EUROPEA (2007c). «Reglamento (CE) n. ${ }^{\circ} 616 / 2007$ de la Comisión de 4 de junio de 2007 relativo a la apertura y modo de gestión de contingentes arancelarios comunitarios en el sector de la carne de aves de corral originaria de Brasil, Tailandia y otros terceros países». Diario oficial, n.ํㄴ L142 de 5 de junio de 2007, pp. 3-11. Disponible en: https:// eur-lex.europa.eu/search.html?qid $=152838$ 2582690\&text $=616 / 2007 \&$ scope $=E U R L E X$ \&type $=q u i c k \& l a n g=e s$

[17] UNIÓN EUROPEA (2008a). «Reglamento (CE) n.. 1296/2008 de la Comisión, de 18 
de diciembre de 2008, por el que se establecen disposiciones de aplicación de los contingentes arancelarios por importación de maíz y de sorgo en España y de maíz en Portugal». Diario oficial, n.으 L340 de 19 de diciembre de 2008. Disponible la última versión consolidada en: https://eur-lex.europa. eu/legal-content/ES/TXT/PDF/?uri=CELEX: 02008R1296-20180126\&qid $=152837845543$ $4 \&$ from $=E S$

[18] UNIÓN EUROPEA (2008b). «Reglamento (CE) n.․ 431/2008 de la Comisión de 19 de mayo de 2008 relativo a la apertura y modo de gestión de un contingente arancelario de importación de carne de vacuno congelada del código NC 0202 y productos del código NC 020629 91 ». Diario oficial, n.으 L130 de 20 de mayo de 2008. Disponible en: https://eurlex.europa.eu/legal-content/ES/TXT/?qid= 1528446609550\&uri=CELEX:02008R043120170101

[19] UNIÓN EUROPEA (2009). «Reglamento (CE) n. ${ }^{\circ}$ 610/2009 de la Comisión de 10 de julio de 2009 por el que se establecen las disposiciones de aplicación del contingente arancelario de carne de vacuno originaria de Chile». Diario oficial, n.. L180 de 11 de julio de 2009, pp. 5-14. Disponible en: $h$ ttps://eur-lex.europa. eu/legal-content/ES/TXT/? qid= 1528446558496\&uri=CELEX:02009R061020130101

[20] UNIÓN EUROPEA (2010). «Acuerdo en forma de Canje de Notas entre la Comunidad Europea y la República Árabe de Egipto sobre medidas recíprocas de liberalización del comercio de productos agrícolas, productos agrícolas transformados y pescado y productos de la pesca, sustitución de los Protocolos n. .1 y n.. 2 y sus anexos, y modificación del Acuerdo euromediterráneo por el que se establece una asociación entre las Comunidades Europeas y sus Estados miembros, por una parte, y la República Árabe de Egipto, por otra». Diario oficial, n. ${ }^{\circ}$ L106 de 24 de abril de 2010, pp. 41-56.

[21] UNIÓN EUROPEA (2011). «Reglamento de ejecución (UE) n.ำ 1273/2011 de la Comisión de 7 de diciembre de 2011 relativo a la apertura y el modo de gestión de determinados contingentes arancelarios de importación de arroz y arroz partido». Diario oficial, n.ㄴ L325 de 8 de diciembre de 2011, pp. 6-23. Disponible en: https://eur-lex.europa.eu/legal-content/ $E S / T X T /$ ?qid $=1532940198152 \&$ uri $=$ CELEX: 02011R1273-20130701

[22] UNIÓN EUROPEA (2012). «Reglamento de ejecución (UE) n.․ 481/2012 de la Comisión de 7 de junio de 2012 por el que se establecen las normas de gestión de un contingente arancelario de carne de vacuno de calidad superior». Diario oficial, n.. $\mathrm{L} 148$ de 8 de junio de 2012, pp. 9-14. Disponible en: https://eur-lex. europa.eu/legal-content/ES/TXT/?qid= 1528382630761\&uri=CELEX:32012R0481

[23] UNIÓN EUROPEA (2013a). «Reglamento (UE) N N. $.1308 / 2013$ del Parlamento Europeo y del Consejo de 17 de diciembre de 2013 por el que se crea la organización común de mercados de los productos agrarios y por el que se derogan los Reglamentos (CEE) n.ㅇ 922/72, (CEE) n.․ 234/79, (CE) n.․ 1037/2001 y (CE) n. $1234 / 2007$ ». DOUE, L347 de 20 de diciembre de 2013, pp. 671-854. Disponible en: https://eur-lex.europa.eu/legal-content/ES/ $T X T /$ ? qid $=1527249954345 \&$ uri $=$ CELEX: 32013R1308

[24] UNIÓN EUROPEA (2013b). «Reglamento (UE) n.. 952/2013 del Parlamento Europeo y del Consejo, de 9 de octubre de 2013, por el que se establece el código aduanero de la Unión». DOUE, $\mathrm{n} .{ }^{\circ} \mathrm{L} 269$ de 10 de octubre de 2013, pp. 1-101. Disponible en: https://eur-lex. europa.eu/legal-content/ES/TXT/?uri=celex \%3A32013R0952

[25] UNIÓN EUROPEA (2013c). «Reglamento de ejecución (UE) n. 593/2013 de la Comisión de 21 de junio de 2013 relativo a la apertura y el modo de gestión de los contingentes arancelarios de carnes de vacuno de calidad superior fresca, refrigerada o congelada, y de carne de búfalo congelada». Diario oficial n. ${ }^{-}$L170, de 22 de junio de 2013, pp. 32-42. Disponible en: https://eur-lex.europa.eu/legalcontent/ES/TXT/?qid $=1528382685579$ \&uri $=$ CELEX:32013R0593

[26] UNIÓN EUROPEA (2014). «Acuerdo de Asociación entre la Unión europea y sus Estados miembros, por una parte, y Ucrania, por otra». Diario oficial, n. ${ }^{\circ} \mathrm{L} 161$ de 29 de mayo de 2014. 
Subdirección General de Comercio Internacional de Productos Agroalimentarios

[27] UNIÓN EUROPEA (2015a). Reglamento de ejecución (UE) 2015/2077 de la Comisión de 18 de noviembre de 2015 relativo a la apertura y modo de gestión de contingentes arancelarios de importación de la Unión de huevos, ovoproductos y albúminas originarios de Ucrania». Diario oficial, n.․ 302 de 19 de noviembre de 2015, pp. 57-62. Disponible en: https://eur-lex.europa.eu/legal-content/ES/ $T X T /$ ? qid $=1530616583028 \&$ uri $=$ CELEX: $32015 R 2077$

[28] UNIÓN EUROPEA (2015b). «Reglamento de Ejecución (UE) 2015/2447 de la Comisión de 24 de noviembre de 2015 por el que se establecen normas de desarrollo de determinadas disposiciones del Reglamento (UE) n. $.952 / 2013$ del Parlamento Europeo y del Consejo por el que se establece el código aduanero de la Unión». DOUE, n. ${ }^{\circ}$ L343 de 29 de diciembre de 2015, pp. 558-893. Disponible en: https://eur-lex.europa.eu/legal-content/ES/TXT/?qid $=1527249675522$ \&uri=CELEX:32015R2447

[29] UNIÓN EUROPEA (2016a). «Reglamento de ejecución (UE) 2016/1239 de la Comisión de 18 de mayo de 2016 por el que se establecen disposiciones de aplicación del Reglamento (UE) n. ${ }^{\circ}$ 1308/2013 del Parlamento Europeo y del Consejo en lo relativo al régimen de certificados de importación y exportación». Diario oficial, n. ${ }^{\circ}$ L206 de 30 de julio de 2016. Disponible en: https://eur-lex.europa.eu/legal-content/ES/TXT/?qid $=1530616423145$ \&uri=CELEX:02016R1239-20171029

[30] UNIÓN EUROPEA (2016b). «Acuerdo de Asociación Económica entre la Unión Europea y sus Estados miembros, por una parte, y los Estados del AAE de la SADC, por otra». Diario oficial, n.․․ L250 de 16 de septiembre de 2016.

[31] UNIÓN EUROPEA (2017a). «Acuerdo Económico y Comercial Global (CETA) entre Canadá, por una parte, y la Unión Europea y sus Estados miembros, por otra». Diario oficial, n.. L 11 de 14 de enero de 2017.

[32] UNIÓN EUROPEA (2017b). «Acuerdo en forma de Canje de Notas entre la Unión Europea e Islandia sobre preferencias comerciales adicionales en el sector de los productos agrícolas». Diario oficial, n.. L274 de 24 de octubre de 2017, pp. 58-201. Disponible en: https://eurlex.europa.eu/legal-content/ES/TXT/?qid= 1528448357352\&uri=CELEX:22017A1024(02)

[33] UNIÓN EUROPEA (2017c). «Decisión (UE) 2017/2423 del Consejo de 11 de diciembre de 2017 relativa a la posición que se debe adoptar en nombre de la Unión Europea en el Consejo de Asociación UE-Turquía por lo que respecta a la modificación del Protocolo n. 02 de la Decisión n.ำ 1/98 del Consejo de Asociación CE-Turquía relativa al régimen comercial aplicable a los productos agrícolas». Diario oficial, n. ${ }^{\circ}$ L343 de 22 de diciembre de 2017, pp. 67-69.

[34] UNIÓN EUROPEA (2018). «Acuerdo en forma de Canje de Notas entre la Unión Europea y el Reino de Noruega sobre preferencias comerciales adicionales en el sector de los productos agrícolas". Diario oficial, n. ${ }^{\circ}$ L129 de 25 de mayo de 2018, pp. 3-15. Disponible en: https:// eur-lex.europa.eu/legal-content/ES/TXT/? uri=uriserv:OJ.L_.2018.129.01.0003.01.SPA \&toC=OJ:L:2018:129:TOC 